\title{
Ruderman-Kittel-Kasuya-Yosida exchange interaction in many-valley IV-VI semimagnetic semiconductors
}

\author{
T. Story, ${ }^{*}$ P. J. T. Eggenkamp, C. H. W. Swüste, H. J. M. Swagten, and W. J. M. de Jonge \\ Department of Physics, Eindhoven University of Technology, 5600 MB Eindhoven, The Netherlands \\ L. F. Lemmens \\ Institute for Applied Mathematics, University of Antwerp (RUCA), Groenenborgerlaan 171, B-2020 Antwerpen, Belgium
}

(Received 11 June 1991)

\begin{abstract}
The Ruderman-Kittel-Kasuya-Yosida (RKKY) indirect-exchange interaction via free carriers is analyzed in the case of IV-VI semimagnetic semiconductors (diluted magnetic semiconductors). Carriers responsible for the RKKY interaction in these materials originate from the anisotropic band of heavy holes located at the $\Sigma$ point of the Brillouin zone (i.e., there are 12 equivalent valleys of this band). Both intervalley and intravalley electron processes contribute to the exchange coupling. Calculations of the RKKY exchange integral and the paramagnetic Curie temperature $(\Theta)$ are presented. The exchange integral is anisotropic, and its dependence on the interspin distance is significantly modified in such a way that the role of antiferromagnetic couplings is increased. As a consequence, the value of $\Theta$ is reduced.
\end{abstract}

\section{INTRODUCTION}

In this paper we will present an analysis of the Ruderman-Kittel-Kasuya-Yosida ${ }^{1}$ (RKKY) indirectexchange interaction via free carriers in IV-VI semimagnetic semiconductors (diluted magnetic semiconductors). ${ }^{2}$ These materials are substitutional solid solutions of the well-known IV-VI semiconductors (such as, e.g., $\mathrm{PbTe}$ or $\mathrm{SnTe}$ ) and $\mathrm{MnTe}$. In the experimentally studied samples we usually have a small amount $(x \leq 0.1)$ of $\mathrm{Mn}$ ions. The results of magnetic measurements [electron paramagnetic resonance 3 (EPR), magnetization, and magnetic susceptibility ${ }^{4-10}$ ] provide experimental evidence that $\mathrm{Mn}$ incorporates into the IV-VI semiconducting matrix as a $\mathrm{Mn}^{2+}$ ion with localized spin moment $\left(S=\frac{5}{2}\right.$, $g=2.0$ ).

From the point of view of electronic properties, IV-VI diluted magnetic semiconductors (DMS) have a metallic type of conductivity with a very large, temperatureindependent, concentration of carriers. Carriers are generated by metal sublattice vacancies, and their concentration may be controlled by thermal annealing. IV-VI materials are narrow-gap semiconductors. The band of electrons and the band of light holes are separated by a direct gap at the $L$ point of the Brillouin zone. In $p$-type crystals with a very large concentration of holes the second valence band of heavy holes is also populated [Fig. 1(a)]. The top of this band is located at the $\Sigma$ point of the Brillouin zone and therefore there are 12 equivalent energy valleys of this band.

The IV-VI DMS present a broad spectrum of magnetic properties. Crystals with relatively low concentration of carriers (e.g., in $\mathrm{Pb}_{1-x} \mathrm{Mn}_{x}$ Te usually $n, p \leq 10^{19} \mathrm{~cm}^{-3}$ ) are paramagnets, ${ }^{10}$ and a spin-glass phase was observed in $\mathrm{Pb}_{1-x} \mathrm{Mn}_{x} \mathrm{Te}$ at very low temperatures $T<1 \mathrm{~K} .{ }^{6}$ Magnetic moments in these crystals are weakly antiferromagnetically coupled via the superexchange interac- tion. Other interspin interaction mechanisms (e.g., direct exchange or the RKKY interaction) are expected to be negligible due to the large mean interspin distances and low concentration of carriers. Crystals with very high concentration of carriers (in $\mathrm{Sn}_{1-x} \mathrm{Mn}_{x} \mathrm{Te}$ and $\mathrm{Ge}_{1-x} \mathrm{Mn}_{x}$ Te usually $p \cong 10^{21} \mathrm{~cm}^{-3}$ ) are ferromagnetic at low temperatures. ${ }^{4-10}$ In this case it is believed that the RKKY interaction via free holes is responsible for

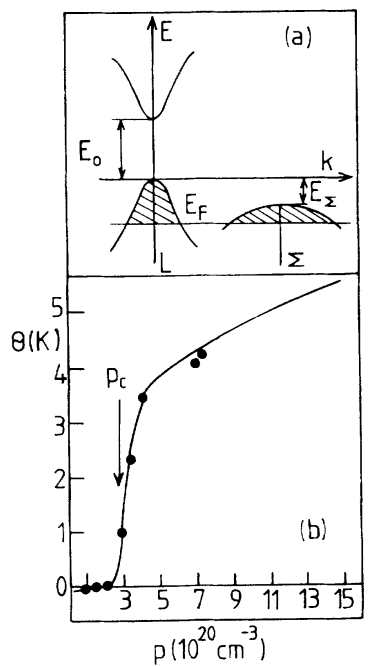

FIG. 1. A simple model of the band structure of the IV-VI semimagnetic semiconductors (a). The band of heavy holes $(\Sigma)$ starts to be populated for concentration of carriers $p>p_{c}$ defined as $E_{F}\left(p_{c}\right)=E_{\Sigma}$. Due to the very high effective mass of $\Sigma$ carriers the RKKY mechanism is strongly enhanced, which results in a thresholdlike carrier concentration dependence of the Curie temperature (b). The experimental data were obtained for the samples of $\mathrm{Pb}_{0.25} \mathrm{Sn}_{0.72} \mathrm{Mn}_{0.03} \mathrm{Te}$ (Ref. 7). The solid line is the theoretical curve based on the calculations of the RKKY interaction (Ref. 8). 
the coupling of the magnetic moments. The transition from the first (paramagnetic) type of behavior to the second (ferromagnetic) one has been observed for certain compositions of $\mathrm{Pb}_{1-x-y} \mathrm{Sn}_{y} \mathbf{M n}_{x} \mathrm{Te}$ crystals including $\mathrm{Sn}_{1-x} \mathrm{Mn}_{x}$ Te. $^{7,8,11,12}$ Samples with a carrier concentration $p<p_{c}=3 \times 10^{20} \mathrm{~cm}^{-3}$ are paramagnets, whereas samples with carrier concentration $p>p_{c}$ are ferromagnets. The Curie temperature of these crystals depends on the carrier concentration in a thresholdlike way [Fig. 1(b)]. ${ }^{7-9}$ The existence of such a threshold concentration of carriers can be understood within the two-valenceband model of the band structure of IV-VI semiconductors. ${ }^{9}$ The threshold concentration $\left(p_{c}\right)$ equals the number of carriers necessary to start to fill the band of heavy holes. Due to the very large density-of-states effective mass of these carriers, the RKKY interaction is significantly enhanced and strong enough to establish a ferromagnetic phase at low temperatures. For samples with $p<p_{c}$ only the light-hole band is populated, which yields a strongly reduced RKKY mechanism.

The strong carrier concentration dependence of the magnetic properties of $\mathrm{Pb}_{1-x-y} \mathrm{Sn}_{y} \mathrm{Mn}_{x} \mathrm{Te}$ crystals and the existence of a ferromagnetic state for rather low concentration of magnetic moments $(x>0.5$ at. \%) strongly suggests that the long-range RKKY indirect-exchange interaction through carriers is the most likely physical mechanism responsible for the exchange coupling of $\mathrm{Mn}$ spins in IV-VI semimagnetic semiconductors with very high concentrations of carriers. These carriers originate from the band of heavy holes located at the $\Sigma$ point of the Brillouin zone. In the present paper we will analyze the RKKY interaction due to these carriers with a special emphasis on the role of anisotropy and many-valley structure of this band. We will show that in addition to the standard intravalley electronic transitions one has also to take into account the intervalley transitions. It will be shown that these transitions contribute about $30 \%$ of the total exchange interaction as measured by the paramagnetic Curie temperature $(\Theta)$. An important modification of the interspin distance dependence of the RKKY exchange integral is also observed.

\section{BAND STRUCTURE}

A band-structure model based on the consistent interpretation of transport, optical, and magnetic experimental data is presented in Fig. $1(\mathrm{a}){ }^{8,9,13}$ The main energy gap is located at the $L$ point of the Brillouin zone. There are four equivalent valleys of both the band of electrons and the band of light holes. These bands are anisotropic ( $A_{L}=m_{\|} / m_{\perp} \cong 10$ in IV-VI tellurides). ${ }^{14}$ Due to the narrow energy gap the energy dispersion relation is nonparabolic and usually described within the Dimmock model. ${ }^{14}$ Approximately $E_{\Sigma}=0.2 \mathrm{eV}$ below the top of the band of light holes there is a second valence band of heavy holes, which is expected to be parabolic. ${ }^{15}$ Significant anisotropy of the Fermi surface is theoretically predicted ${ }^{15}$ and experimentally observed. ${ }^{16-18}$ The value of the effective mass of carriers in the $\Sigma$ band, estimated from the optical measurements, ${ }^{13}$ is very large, $m_{\Sigma} \cong 1.7 m_{0}$. In our calculations we will use the value of the effective mass anisotropy coefficient of heavy holes $A_{\Sigma}=10$ with the Fermi surface in a form of an ellipsoid of revolution. ${ }^{16-18}$ From theoretical band-structure calculations, ${ }^{14}$ it follows that the band of heavy holes is located approximately halfway from the center of the zone to the edge of the first Brillouin zone into the [110] direction, i.e., point $K[3 \pi / 2 a ; 3 \pi / 2 a ; 0]$. Here $a=6.33 \AA$ is the typical value of the lattice constant of the rocksalt crystal lattice of IV-VI semiconductors. The allocation of these valleys in the Brillouin zone is presented in Fig. 2. ${ }^{15,16}$ The dimensions of the Fermi ellipsoids are smaller than the dimensions of the first Brillouin zone and all 12 ellipsoids are located within the first zone. For carrier concentrations realized in the experimental studies $\left(p \leq 10^{21} \mathrm{~cm}^{-3}\right)$ they also are not connected. For each of the 12 Fermi ellipsoids the energy dispersion relation has the following form:

$E_{n}(k)=\frac{\hbar^{2}}{2}\left(\frac{\left(k_{x}-K_{n x}\right)^{2}}{m_{\perp}}+\frac{\left(k_{y}-K_{n y}\right)^{2}}{m_{\perp}}+\frac{\left(k_{z}-K_{n z}\right)^{2}}{m_{\|}}\right)$,

where $\mathbf{K}_{n}$ determines the position of the center of a given valley (Fig. 2). The shape of the Fermi ellipsoids $\left(k_{F \|}\right.$, $k_{F \perp}$ in Fig. 3) is determined by the concentration of carriers and the effective-mass anisotropy coefficient $A$ :

$$
k_{F \|}=\left(3 \pi^{2} \widetilde{p}\right)^{1 / 3} A^{1 / 3}, \quad k_{F \perp}=\left(3 \pi^{2} \widetilde{p}\right)^{1 / 3} A^{-1 / 6} .
$$

Here $\widetilde{p}=p / 12$ is the concentration of carriers in each valley. In the case of an isotropic band ( $A=1)$, Eq. (2) gives the standard result: $k_{F \|}=k_{F \perp}=\left(3 \pi^{2} \tilde{p}\right)^{1 / 3}$.

The carriers at these Fermi surfaces are almost entirely responsible for the RKKY exchange mechanism in IV-VI crystals. In the next section we will analyze the effect of the many-valley structure and anisotropy of the Fermi surface of carriers on the RKKY exchange mechanism.

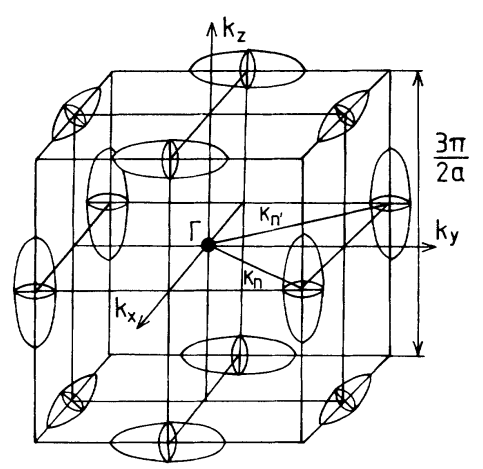

FIG. 2. The allocation of 12 Fermi ellipsoids of the band of heavy holes. The ellipsoids are located at the $\Sigma$ points of the Brillouin zone and prolonged into the [100] directions. This model was developed based on the experimental measurements of magnetoresistance (Ref. 16) and the de Haas-van Alphen effect (Ref. 18). 


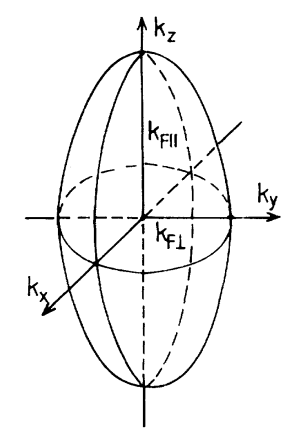

FIG. 3. The Fermi surface of the $\Sigma$ carriers has the shape of the ellipsoid of revolution with the long axis along one of the [100] directions. The dimensions of the Fermi ellipsoids are determined by the concentration of carriers and the effectivemass anisotropy coefficient [Eq. (2)].

\section{RKKY INTERACTION}

The general expression for the RKKY exchange integral $I\left(\mathbf{R}_{i j}\right)$ can be derived based on the second-order perturbation theory with the perturbing potential $\hat{\mathbf{H}}=J_{s d} \widehat{\mathbf{S}} \cdot \hat{\boldsymbol{\sigma}}$. This Hamiltonian describes the interaction between localized magnetic moments $\widehat{\mathbf{S}}$ and free carriers $\hat{\sigma}$ by $s-d$ exchange. As a result one obtains the spindependent second-order correction to the energy of the magnetic subsystem: ${ }^{1,19} E=-I_{i j} \widehat{\mathbf{S}}_{i} \cdot \widehat{\mathbf{S}}_{j}$, with the RKKY exchange integral $I_{i j}$ :

$$
I\left(\mathbf{R}_{i j}\right)=\frac{1}{2} \sum_{\alpha, \alpha^{\prime}} \sum_{k, k^{\prime}}\left(\frac{f_{\alpha k}-f_{\alpha^{\prime} k^{\prime}}}{E_{\alpha^{\prime}}\left(k^{\prime}\right)-E_{\alpha}(k)}\right) J_{s d}^{2} \exp \left(i \mathbf{q} \cdot \mathbf{R}_{i j}\right) .
$$

Here $\alpha$ labels electron bands, $k$ and $k^{\prime}$ are the wave vectors of carriers, $f_{\alpha k}$ is the Fermi-Dirac distribution function, and $E_{\alpha}(k)$ the energy of the carrier in band $\alpha$. The vector $R_{i j}$ denotes the position of the spins in the crystal lattice and vector $q$ is defined as $q=\mathbf{k}-\mathbf{k}^{\prime}$.

Microscopically, the RKKY exchange interaction can be considered as a second-order scattering process, ${ }^{1}$ where the electron in the quantum state $|\alpha \mathbf{k}\rangle$ is scattered by the magnetic moment into the intermediate state $\left|\alpha^{\prime} \mathbf{k}^{\prime}\right\rangle$ and subsequently scattered again by the other magnetic moment to the initial state $|\alpha \mathbf{k}\rangle$. The $s-d$ exchange integral $J_{s d}=J\left(\alpha \mathbf{k}, \alpha^{\prime} \mathbf{k}^{\prime}\right)$ (the matrix element determining the coupling of electron states $\alpha k$ and $\alpha^{\prime} k^{\prime}$ ) depends generally on $\mathbf{k}$ and $\mathbf{k}^{\prime} .{ }^{20}$ In the spirit of standard RKKY calculations ${ }^{1}$ we will neglect this dependence and treat the $J_{s d}$ as a constant. Physically, it means that the local character of the $s-d$ exchange interaction is approximated by a $\delta$ function.

In the case of IV-VI crystals there is no band summation in Eq. (3) since carriers from only one band dominate the RKKY exchange mechanism. The sum over $k, k^{\prime}$ is restricted to the first Brillouin zone. Every possible choice of the wave vectors $k, k^{\prime}$ has to be taken into account. Direct inspection of Eq. (3) leads to the conclusion that there is no contribution to the exchange integral $I\left(\mathbf{R}_{i j}\right)$ due to electron processes in which both $k$ and $k^{\prime}$ are located inside $\left(f_{k}=f_{k^{\prime}}=1\right)$ or outside $\left(f_{k}=f_{k^{\prime}}=0\right)$ the Fermi ellipsoids. The largest contribution is expected in the situation where $k$ is located just below the Fermi surface and $k^{\prime}$ is just above this surface (see Fig. 4). In this case one has $f_{k}-f_{k^{\prime}} \cong 1$ and $E\left(k^{\prime}\right)-E(k) \cong 0$. These are standard intravalley electron transitions responsible for the RKKY interaction. One can see, however, that in the many-valley systems a large contribution to the RKKY exchange integral may also be generated by intervalley transitions. In this case vector $k$ is located just below the Fermi surface of the $n$th valley whereas $k^{\prime}$ is just above the Fermi surface of the other $n^{\prime}$ th valley (Fig. 4). A difference between these two cases may be caused by a (possibly) different value of the $s-d$ exchange constant $J_{s d}$ due to large $q$ transitions in the case of intervalley processes. However, as mentioned before, within the frame of the standard RKKY calculations the $J_{s d}$ exchange integral does not depend on the $\mathbf{q}=\mathbf{k}-\mathbf{k}^{\prime}$ and both types of transitions are weighted in the same way.

Based on the above discussion we can conclude that the problem of the summation over the whole first Brillouin zone reduces to the summation over the spectrum of all possible pairs of valleys (both $n=n^{\prime}$ and $n \neq n^{\prime}$ ) with $k \leq k_{n F}$ and $k^{\prime} \geq k_{n^{\prime} F}$ (and vice versa).

Below we will calculate the contribution to the RKKY exchange integral due to the electron transitions between two valleys (Fig. 5). To perform the summation (3) we will introduce new $k$-space coordinates:

$$
\begin{array}{ll}
\kappa_{x}=\left(k_{x}-K_{n x}\right) m_{\perp}^{-1 / 2}, & \kappa_{x}^{\prime}=\left(k_{x}^{\prime}-K_{n^{\prime} x}\right) m_{\perp}^{-1 / 2}, \\
\kappa_{y}=\left(k_{y}-K_{n y}\right) m_{\perp}^{-1 / 2}, & \kappa_{y}^{\prime}=\left(k_{y}^{\prime}-K_{n^{\prime} y}\right) m_{\perp}^{-1 / 2}, \\
\kappa_{z}=\left(k_{z}-K_{n z}\right) m_{\|}^{-1 / 2}, & \kappa_{z}^{\prime}=\left(k_{z}^{\prime}-K_{n^{\prime} z}\right) m_{\|}^{-1 / 2} .
\end{array}
$$

The energy dispersion relation now has the parabolic form

$$
E_{n}(k)=\frac{1}{2} \hbar^{2} \kappa^{2} .
$$

We will also use the new $R$-space coordinates:

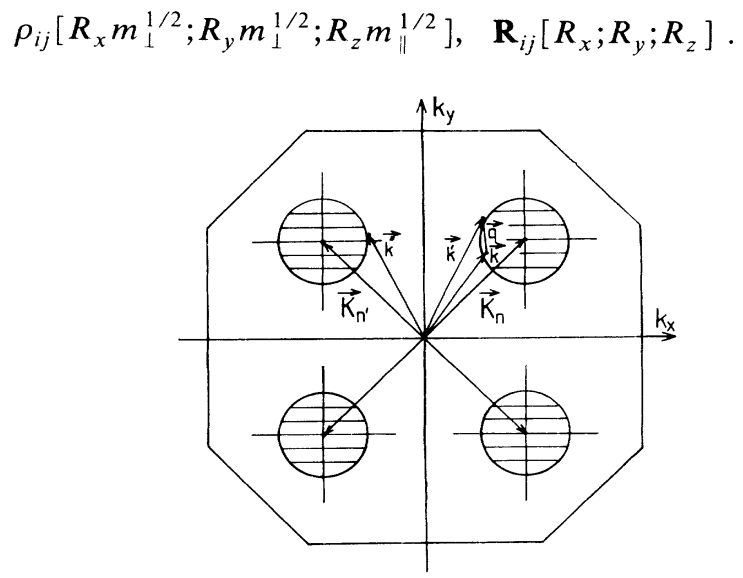

FIG. 4. The $k_{x}-k_{y}$ cross section of the first Brillouin zone of the IV-VI crystals. The pair of wave vectors $\left(\mathbf{k}, \mathbf{k}^{\prime}\right)$ represents the standard electronic transitions responsible for the RKKY interaction. The pair $\left(\mathbf{k}, \mathbf{k}^{\prime \prime}\right)$ represents the intervalley transitions present in many-valley crystals. 


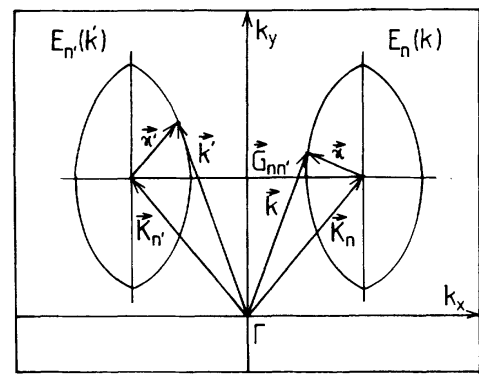

FIG. 5. The pair of two ellipsoids prolonged into the same direction. In such a case the anisotropic problem of the RKKY interaction calculations can be reduced to the isotropic one via transformation (4)-(7). The energy of carriers in valley $n$ is given by $E_{n}(k)=\left(\hbar^{2} / 2\right) \kappa^{2}$.

The old and new variables are related via Eq. (7):

$$
\mathbf{q} \cdot \mathbf{R}_{i j}=\left(\mathbf{k}-\mathbf{k}^{\prime}\right) \cdot \mathbf{R}_{i j}=\left(\boldsymbol{\kappa}-\boldsymbol{\kappa}^{\prime}\right) \cdot \boldsymbol{\rho}_{i j}+\left(\mathbf{K}_{n}-\mathbf{K}_{n^{\prime}}\right) \cdot \mathbf{R}_{i j} .
$$

With these new variables and after transformation of expression (3) into an integral form, the RKKY exchange integral may be written as follows:

$$
\begin{aligned}
I\left(\mathbf{R}_{i j}\right)=\frac{V m_{\perp}^{2} m_{\|}}{8 \pi^{3} \hbar^{2}} \int & d^{3} \kappa d^{3} \kappa^{\prime}\left(\frac{f_{n \kappa}-f_{n^{\prime} \kappa^{\prime}}}{\kappa^{\prime 2}-\kappa^{2}}\right) J_{s d}^{2} \\
& \times \exp \left(i \mathbf{q}^{\prime} \cdot \rho_{i j}\right) \exp \left(i \mathbf{G}_{n n} \cdot \mathbf{R}_{i j}\right)
\end{aligned}
$$

where $\mathrm{q}^{\prime}=\boldsymbol{\kappa}-\boldsymbol{\kappa}^{\prime}, V$ is the volume of the crystal, and $\mathbf{G}_{n n^{\prime}}=\mathbf{K}_{n}-\mathbf{K}_{n^{\prime}}$, is the separation vector of a pair of valleys.

The integral in Eq. (8) is the standard RKKY expression with an additional constant phase factor $\exp \left(i \mathbf{G}_{n n^{\prime}} \cdot \mathbf{R}_{i j}\right)$. Integration of (8) yields

$$
I\left(\mathbf{R}_{i j}\right) A F_{\mathbf{R K}}\left(z=2 \kappa_{F} \rho\right) \cos \left(\mathbf{G}_{n n^{\prime}} \cdot \mathbf{R}_{i j}\right),
$$

where

$$
A=\frac{1}{32 \pi^{3} \hbar^{2}} J_{s d}^{2} a^{6} m_{\perp}^{2} m_{\|} \kappa_{F}^{4} .
$$

Here $F_{\mathrm{RK}}(z)=[\sin (z)-z \cos (z)] / z^{4}$ is the Ruderman-Kittel function. The Fermi wave vector $k_{F}$ and the interspin distance $R_{i j}$ present in the standard RKKY result are now replaced by reduced quantities $\kappa_{F}$ and $\rho$ defined below:

$$
\begin{aligned}
& \kappa_{F}^{2}=\frac{2 E_{F}}{\hbar^{2}}=\frac{k_{F x}^{2}+k_{F y}^{2}}{m_{\perp}}+\frac{k_{F z}^{2}}{m_{\|}}, \\
& \rho^{2}=R_{x}^{2} m_{\perp}+R_{y}^{2} m_{\perp}+R_{z}^{2} m_{\|} .
\end{aligned}
$$

The reduced Fermi wave vector $\kappa_{F}$ is related to the half axes of the Fermi ellipsoids:

$$
\kappa_{F}=\frac{k_{F 1}}{m_{\perp}^{1 / 2}}=\frac{k_{F \|}}{m_{\|}^{1 / 2}} \text {. }
$$

Based on Eqs. (11) and (12) the argument $(z)$ of the
Ruderman-Kittel function can be expressed as follows:

$$
\begin{aligned}
z=2 \kappa_{F} \rho= & 2 k_{F 1} R_{A z}, \\
& \text { where } R_{A z}=\left(R_{x}^{2}+R_{y}^{2}+A R_{z}^{2}\right)^{1 / 2} .
\end{aligned}
$$

Here $k_{F \perp}$ is given by Eq. (2).

One can see that the RKKY exchange integral [Eq. (9)] is again described by the Ruderman-Kittel function $F_{\mathrm{RK}}(z)$. As a consequence of the anisotropic Fermi surface the argument $(z)$ of $F_{\mathrm{RK}}$ is now also anisotropic causing the anisotropy of the exchange integral.

The result given by Eqs. (9)-(13) was obtained assuming that the main axes of the Fermi ellipsoids coincide with the cubic cystallographic directions and the ellipsoids are prolonged into the $k_{z}[001]$ direction. If this is not the case one has to transform the result from the coordinate system related to the main axes of the Fermi ellipsoids to the cubic crystal axes within which the location of spins is described. This can be done using a standard transformation matrix. As a result the expression (13) for the argument of the $F_{\mathrm{RK}}$ function will have a modified form. In our case all the valleys prolonged into the $k_{z}$ direction are in fact located as described above. Due to the circular symmetry of the Fermi surface in the $k_{x}-k_{y}$ plane the specific choice of the $k_{x}, k_{y}$ axes is not important. In the case of ellipsoids prolonged into $k_{x}[100]$ or $k_{y}[010]$ direction one can perform the transformation with the following result:

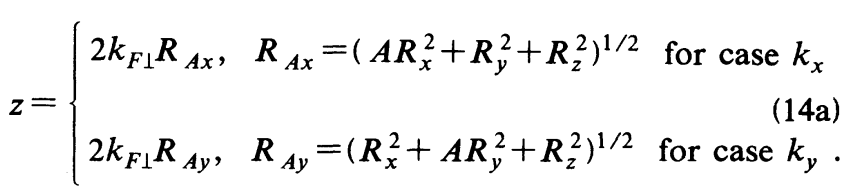

The result (14a), (14b) has a simple interpretation. The cases $k_{x}$ and $k_{y}$ are in fact equivalent to the $k_{z}$ one. The role of "special" coordinate is played by $x, y$, or $z$ depending on the direction into which the ellipsoids are prolonged. This very simple result is valid only in the case of Fermi surfaces in the form of ellipsoids of revolution with the long axis prolonged along one of the [100] directions.

The spectrum of different pairs of valleys (see Table I and Fig. 2) also contains the pairs prolonged into two orthogonal directions. In this situation the expression (3) cannot be reduced to the standard form [Eqs. (8)-(10)] via transformation of $R$ and $k$ space [Eqs. (4)-(7)] and calculations can only be performed numerically. We will approximate these contributions by an arithmetic mean value of two relevant parallel pairs [see Eq. (17b)].

In the simple case of one isotropic valley $\left(n=n^{\prime}\right)$ the phase factor reduces to unity $\left(G_{n n^{\prime}}=0\right)$ and the argument $z=2 \kappa_{F} \rho=2 k_{F} R$, i.e., we have recovered the standard result of Ruderman and Kittel.

In the model case of one anisotropic valley the phase factor is also not present $\left(G_{n n^{\prime}}=0\right)$ but the result is generally anisotropic and equal to the results of model calculations of Baltensperger and de Graaf ${ }^{21}$ and Lara, Moreira Xavier, and Taft. ${ }^{22}$ The surface of equal interaction $\left[I\left(\mathbf{R}_{i j}\right)=\right.$ const $]$, which in the standard case is spher- 
ical, now has the form of an ellipsoid of revolution. The prolonged axis of this ellipsoid coincides with the crystal direction characterized by the small (i.e., $m_{\perp}$ ) effective mass.

In the case of one pair of isotropic valleys we have the result obtained by Cullen, Callen, and Luther ${ }^{23}$ and Kit$\mathrm{tel}^{24}$ for such a model situation:

$$
I\left(\mathbf{R}_{i j}\right)=2 I_{0}\left[1+\cos \left(\mathbf{G}_{12} \cdot \mathbf{R}_{i j}\right)\right],
$$

where $I_{0}$ is the standard one-valley contribution given by Eqs. (9) and (10) in the isotropic case.

One can see that one pair of valleys generates $2^{2}$ contributions to the RKKY exchange integral: two (equal) intravalley terms $I_{0}$ and two (equal) intervalley terms $I_{0} \cos \left(\mathbf{G}_{12} \cdot \mathbf{R}_{i j}\right)$. The presence of the cosine factor in Eq. (15) introduces additional short-range oscillations (usually $\left.k_{F} \ll<G_{12}\right)$ to the distance dependence of $I\left(\mathbf{R}_{i j}\right)$.

Based on the analysis presented above one can conclude that in order to calculate the RKKY exchange integral in an $N$-valley system one has to take into account $N^{2}$ terms, $N$ of which are standard intravalley terms and the rest originating from intervalley transitions. To perform actual calculations one has to analyze the location of valleys in the Brillouin zone (i.e., the spectrum of separation vectors $\mathbf{G}_{n n^{\prime}}$ ) and the direction into which they are prolonged.

The spectrum of all possible pairs of valleys in the case of the $\Sigma$ band of IV-VI semiconductors is presented in Table I and Fig. 2. Due to the cubic symmetry of allocation the total number of different types of pairs is reduced to five. There are 12 intravalley terms $\left(I_{0}\right)$. There are two types of intervalley terms $\left(I_{1}\right.$ and $\left.I_{2}\right)$ in which both Fermi ellipsoids of the pair are parallel. They differ only by different separation vectors $(\mathbf{G})$. There are also two types of intervalley terms of orthogonal pairs $\left(I_{3}\right.$ and $\left.I_{4}\right)$. The representative separation vectors of each term are presented in Table I. Generally, every pair has its own separation vector. Due to the highly symmetrical allocation of the valleys many of the separation vectors are the same.

Using the equations derived above we can calculate the interspin distance dependence of the RKKY exchange in-

TABLE I. The spectrum of pairs of energy valleys in the case of the $\Sigma$ band of IV-VI semiconductors.

\begin{tabular}{|c|c|c|c|}
\hline Term & Type & $\begin{array}{c}\text { Number of } \\
\text { terms }\end{array}$ & $\begin{array}{l}\text { Representative } \\
\text { separation vector }\end{array}$ \\
\hline$I_{0}$ & intravalley & 12 & $\mathbf{G}=0$ \\
\hline$I_{1}$ & $\begin{array}{l}\text { intervalley } \\
\text { parallel }\end{array}$ & 12 & $\mathbf{G}=\frac{3 \pi}{2 a}[110]$ \\
\hline$I_{2}$ & $\begin{array}{l}\text { intervalley } \\
\text { parallel }\end{array}$ & 24 & $\mathbf{G}=\frac{3 \pi}{2 a}[100]$ \\
\hline$I_{3}$ & $\begin{array}{l}\text { intervalley } \\
\text { orthogonal }\end{array}$ & 48 & $\mathbf{G}=\frac{3 \pi}{4 a}[110]$ \\
\hline$I_{4}$ & $\begin{array}{l}\text { intervalley } \\
\text { orthogonal }\end{array}$ & 48 & $\mathbf{G}=\frac{3 \pi}{4 a}[211]$ \\
\hline
\end{tabular}

tegral both with and without the intervalley contributions. For the contribution of the pair $n-n^{\prime}$ of $I_{q}$ type $(q=0,1, \ldots, 4)$ we have the following expression:

$$
I^{(q)}\left(\mathbf{R}_{i j}\right)=A I_{q} \cos \left(\mathbf{G}_{n n^{\prime}} \cdot \mathbf{R}_{i j}\right) \exp \left(-R_{i j} / \lambda\right) .
$$

The factor $\exp \left(-R_{i j} / \lambda\right)$ included in formula (16) takes into account the effect of the reduction of the magnitude of the RKKY interaction due to the short mean free path of carriers $(\lambda) .^{25}$

For all pairs of valleys in which both valleys are prolonged into the same direction $(i)$ (i.e., in the case of $q=0,1,2$ )

$$
I_{q}=F_{\mathrm{RK}}\left(2 k_{F \perp} R_{A i}\right) \text {, where } i=x, y \text {, or } z \text {. }
$$

For all pairs of valleys in which the valleys are prolonged into the two orthogonal directions $(i)$ and $(j)$ (i.e., for $q=3$ and 4)

$$
\begin{array}{r}
I_{q}=\frac{1}{2}\left[F_{\mathrm{RK}}\left(2 k_{F \perp} R_{A i}\right)+F_{\mathrm{RK}}\left(2 k_{F \perp} R_{A j}\right)\right], \\
i, j=x, y, z ; i \neq j .
\end{array}
$$

The total exchange integral is given by the following expression:

$$
I_{\text {total }}\left(\mathbf{R}_{i j}\right)=I_{\text {intra }}\left(\mathbf{R}_{i j}\right)+I_{\text {inter }}\left(\mathbf{R}_{i j}\right),
$$

where $I_{\text {intra }}\left(\mathbf{R}_{i j}\right)$ is the sum of all intravalley contributions and $I_{\text {inter }}\left(\mathbf{R}_{i j}\right)$ is the sum of all intervalley terms.

In the next section we will also calculate the paramagnetic Curie temperature $(\Theta)$ of a model IV-VI semimagnetic crystal. For a randomly diluted magnetic system the paramagnetic Curie temperature can be expressed as

$$
k_{B} \Theta=\frac{2}{3} S(S+1) x \sum_{n} z_{n} I\left(R_{n}\right),
$$

where $z_{n}$ is the number of neighbors on the $n$th crystal shell and $R_{n}=a(n / 2)^{1 / 2}$ is the distance (in the fcc lattice) to the spin located on the $n$th shell, $x$ is the concentration of magnetic ions, and $S$ is the spin magnetic moment of the magnetic impurities ( $S=\frac{5}{2}$ for $\mathrm{Mn}^{2+}$ ). Equation (19) includes the exchange interaction between the reference spin and all the neighbors for which the coupling is not negligible. Implementing (18) in (19) the paramagnetic Curie temperature can also be expressed as a sum of two parts: $\Theta_{\text {total }}=\Theta_{\text {intra }}+\Theta_{\text {inter }}$.

\section{DISCUSSION AND CONCLUSIONS}

The results of calculations of the interspin distance dependence of the RKKY exchange integral according to Eqs. (16) - (18) are presented in Fig. 6. One can compare the standard result [Fig. 6(a)] with the modification caused by the anisotropy of the effective mass of carriers [Fig. 6(b)] and the changes due to the intervalley terms [Fig. 6(c)]. The numerical values presented in Figs. 6 and 7 were obtained for the $\mathrm{Pb}_{0.25} \mathrm{Sn}_{0.72} \mathrm{Mn}_{0.03} \mathrm{Te}$ alloy $\left(a=6.33 \AA, m=1.7 m_{0}, J_{s d}=100 \mathrm{meV}\right) .{ }^{8}$ The mean free path of carriers was assumed to be carrier concentration independent and equal to $\lambda=10 \AA .{ }^{8}$ Calculating the exchange integral in the case of iso- and anisotropic bands the effective mass of the density of states was kept the 


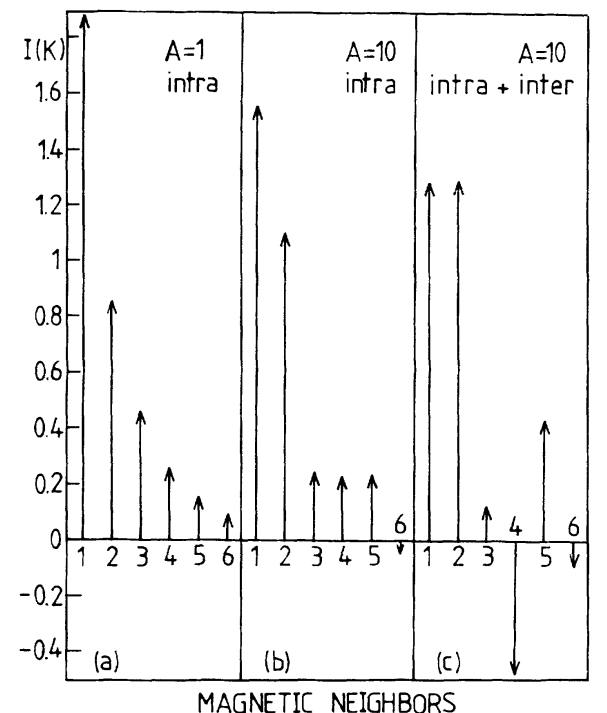

FIG. 6. The interspin distance dependence of the RKKY exchange integral in a model IV-VI semimagnetic alloy. (a) The standard result (isotropic Fermi surface, intravalley electronic transitions). (b) The modification caused by the anisotropy of the Fermi surface $\left(A=m_{\|} / m_{\perp}=10\right)$. The total exchange integral (intra- plus intervalley processes, $A=10$ ).

same. The effect of the anisotropy of the Fermi surface appears to be relatively small [Fig. 6(b)]. The exchange integral, however, is anisotropic now and the spins separated by the same distance but located along the different crystallographic directions are coupled with different strength. For all the equidistant spins located on the cubically equivalent directions (e.g., 12 nearest neighbors) the exchange interaction is the same. Because the $(N+1)$ th neighbors are located on a different direction than the $N$ th neighbors, the directional dependence of the exchange integral may cause a nonmonotonic dependence of the RKKY exchange interaction as a function of distance [Fig. 6(b)].

The effect of the intervalley terms is more significant. Most of the magnetic neighbors are still ferromagnetically coupled to the reference spin. The role of antiferromagnetic couplings is, however, enhanced. For example, the first antiferromagnetic coupling is observed for the fourth neighbor instead of the ninth, as in the standard case. Due to the presence of $\cos \left(\mathbf{G} \cdot \mathbf{R}_{i j}\right)$ factors the total integral is anisotropic even in the case of an isotropic $E(k)$ relation.

The results of calculations of the paramagnetic Curie temperature are presented in Fig. 7. In agreement with the $I(R)$ calculations the effect of anisotropic $E(k)$ is rather small. The effect of the intervalley terms is quite large and it roughly amounts to about $30 \%$ reduction of the value of $\Theta$. The bars presented on the plots in Fig. 7 present the effect caused by a shift of the top of the $\Sigma$ band by $\pm 10 \%$ from the assumed position (halfway from the center of the zone to the $K$ point). The position of the top of the $\Sigma$ band in the Brillouin zone determines the separation vectors of the pairs of valleys and the period of short-range oscillations. It influences, therefore, the

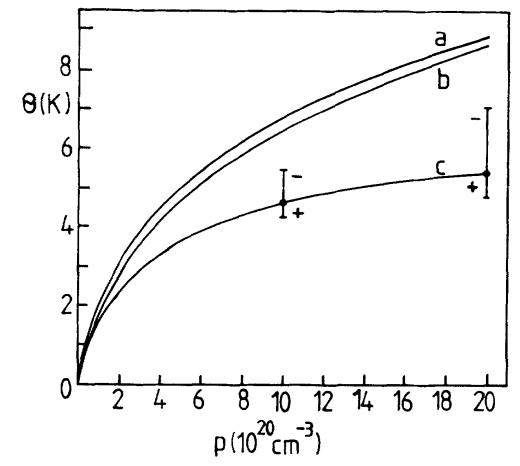

FIG. 7. The carrier concentration dependence of the paramagnetic Curie temperature of a model IV-VI semimagnetic semiconductor $(x=0.03)$. Line $a$, the standard result (isotropic, intravalley). Line $b$, anisotropic Fermi surface, only intravalley electronic transitions. Line $c$, the total paramagnetic $\mathrm{Cu}$ rie temperature (anisotropic Fermi surface, intra- plus intervalley processes).

intervalley part of the paramagnetic Curie temperature $\left(\Theta_{\text {inter }}\right)$ but has no effect on the main intravalley part of $\Theta$.

One can see that, despite the fact that the number of intervalley terms contributing to the total exchange integral is about $N=12$ times larger than the number of intravalley contributions, the latter dominates the total integral. The physical reason of this effect is related to the $\cos (\mathbf{G} \cdot \mathbf{R})$ factors multiplying every intervalley contribution. The resulting coupling between two spins is a sum of a large number of different cosine terms partially canceling each other. In the case of calculations of the paramagnetic Curie temperature an additional cancellation effect is effective. Due to the small period of the oscillations caused by the intervalley transitions $\left(T_{G} \sim 1 / G\right)$, the intervalley contributions have different signs for different neighbors. One can expect the intervalley contribution to the paramagnetic Curie temperature to be very small if the period of RKKY oscillations $\left(T_{K F} \sim 1 / k_{F}\right)$ is much larger as compared to the period related to the intervalley processes $\left(T_{G}\right)$, i.e., $k_{F} \ll G$. The additional condition is that the magnitude of the exchange interaction should decrease relatively slowly with increasing distance providing an efficient cancellation of the intervalley terms.

Finally, a few comments regarding some basic assumptions made in the present analysis must be made. We have already mentioned the assumption that the $s-d$ exchange integral is not dependent on the wave vectors $\mathbf{k}, \mathbf{k}^{\prime}$. The solution of this problem requires the theoretical analysis of the RKKY mechanism taking into account the realistic wave functions of the carriers and $3 d^{5}$ electrons of $\mathrm{Mn}$ ions. One can expect a modification of the model picture of the RKKY mechanism. ${ }^{26}$ To our knowledge such calculations have not been performed yet. There are two other effects expected to influence the RKKY exchange integral. The first is an electronelectron interaction influencing the susceptibility of the electron gas and consequently the RKKY exchange interaction. ${ }^{24}$ This effect was found to be very important 
in, e.g., diluted magnetic systems based on Pd. ${ }^{27}$ This system has an intermediate electron density; i.e., the mean kinetic energy of electrons compares to the mean potential energy. In our system the mean kinetic energy is much larger than the mean potential energy of electrons. This is due to the high dielectric constant which shields the electron-electron interaction, leading to an effective high density system for electronic response. The characteristic $r_{s}$ value, i.e., the Wigner-Seitz volume expressed in units of effective Bohr radius, will be smaller than unity for electron densities above $p \cong 10^{19} \mathrm{~cm}^{-3}$. Therefore we expect that the exchange contribution to the electronic susceptibility will be small. ${ }^{28}$

The second effect is related to the rather small mean free path of the carriers. The phenomenological exponential factor $\exp (-R / \lambda)$ takes into account the decrease of the magnitude of the RKKY interaction. One can also expect the range of the interaction to be reduced. ${ }^{29}$ However, even in the case of small mean free path of carriers observed in IV-VI alloys, this effect is also expected to be rather small. ${ }^{24,29}$

In conclusion, we have investigated the RKKY exchange integral in the case of a model IV-VI semimagnetic semiconductor with anisotropic many-valley structure of the Fermi surface. The anisotropy of the Fermi surface of these semiconductors induces an anisotropy of the
RKKY exchange interaction. The exchange integral depends not only on the interspin distance but also on the crystallographic direction of the vector connecting the interacting spins. The quantitative effect is, however, rather small. In addition to the standard intravalley electronic transitions we also found the intervalley processes to be responsible for the indirect-exchange interaction. The overall contribution, as measured by the paramagnetic Curie temperature, is negative and equal to about $30 \%$ of the total value of $\Theta$. The interspin distance dependence of the exchange integral is significantly modified due to the intervalley transitions in such a way that the role of antiferromagnetic couplings is enhanced. It may have important consequences for the low-temperature magnetic properties of diluted magnetic alloys causing, e.g., the existence of the spin-glass phase to be more likely. ${ }^{30}$

\section{ACKNOWLEDGMENTS}

We would like to acknowledge the stimulating discussions with Professor R. R. Galazka and Dr. A. Twardowski. We would like to thank R. J. H. Groenveld for his assistance in numerical calculations. This work has been financially supported by the Stichting Fundamental Onderzoek der Materie (FOM).
${ }^{*}$ On leave from Institute of Physics, Polish Academy of Sciences, A1. Lotnik øw 32/46, 02-668 Warsaw, Poland.

${ }^{1}$ M. Ruderman and C. Kittel, Phys. Rev. 96, 99 (1954); T. Kasuya, Prog. Theor. Phys. 16, 45 (1956); K. Yosida, Phys. Rev. 106, 893 (1957).

${ }^{2}$ R. R. Galagzka, in Proceedings of the 14th International Conference on the Physics of Semiconductors, Edinburgh, 1978, edited by B. L. H. Wilson, IOP Conf. Proc. No. 43 (Institute of Physics and Physical Society, London, 1978), p. 133; G. Bauer, in Diluted Magnetic Semiconductors, edited by R. L. Aggarwal, J. K. Furdyna, and S. von Molnar (Materials Research Society, Pittsburgh, 1987), Vol. 89, p. 107; Semiconductors and Semimetals, edited by J. K. Furdyna and J. Kossut (Academic, Boston, 1988), Vol. 25.

${ }^{3}$ G. Toth, J. Y. Leloup, and H. Rodot, Phys. Rev. B 1, 4573 (1973); R. W. Cochrane, R. T. Hedgcock, and A. W. Lightstone, Can. J. Phys. 56, 68 (1978); P. Urban and G. Sperlich, Solid State Commun. 16, 927 (1975); S. Z. Korczak, W. Korczak, M. Subotowicz, and H. Wasiewicz, Phys. Status Solidi B 153, 361 (1989).

4J. Cohen, A. Globa, P. Mollard, H. Rodot, and M. Rodot, J. Phys. (Paris) Colloq. 29, C4-143 (1968).

${ }^{5}$ R. W. Cochrane, F. T. Hedgcock, and J. O. Ström-Olsen, Phys. Rev. B 9, 4262 (1973).

${ }^{6} \mathrm{M}$. Escorne, A. Mauger, J. T. Tholence, and R. Triboulet, Phys. Rev. B 29, 6306 (1984).

${ }^{7}$ T. Story, R. R. Galązka, R. B. Frankel, and P. A. Wolff, Phys. Rev. Lett. 56, 777 (1986).

${ }^{8}$ T. Story, G. Karczewski, L. Świerkowski, and R. R. Gałązka, Phys. Rev. B 42, 10477 (1990).

${ }^{9}$ H. J. M. Swagten, W. J. M. de Jonge, R. R. GaYạzka, P. Warmenbol, and J. T. Devreese, Phys. Rev. B 37, 9907 (1988).
${ }^{10}$ M. Górska and J. R. Anderson, Phys. Rev. B 38, 9120 (1988).

${ }^{11}$ T. Story, G. Karczewski, L. Świerkowski, M. Górska, and R. R. GaYagzka, in Proceedings of the International Conference on the Narrow Gap Semiconductors and Related Materials, Gaithersburg, 1989 [Semicond. Sci. Technol. 5, S138 (1990)].

${ }^{12}$ W. J. M. de Jonge, H. J. M. Swagten, S. J. E. A. Eltink, and N. M. J. Stoffels, in Proceedings of the International Conference on Narrow Gap Semiconductors and Related Materials, Gaithersburg, 1989 [Semicond. Sci. Technol. 5, S131 (1990)].

${ }^{13}$ G. Karczewski, L. Swierkowski, T. Story, A. Szczerbakow, J. Niewodniczańska-Blinowska, and G. Bauer, Semicond. Sci. Technol. 5, 1115 (1990).

${ }^{14} \mathrm{G}$. Nimtz and B. Schlicht, in Narrow Gap Semiconductors, Springer Tracts in Modern Physics Vol. 98 (Springer, Berlin, 1983).

${ }^{15}$ O. A. Pankratov and A. V. Sazonov, Fiz. Tverd. Tela (Leningrad) 27, 3506 (1985) [Sov. Phys._Solid State 27, 2117 (1985)].

${ }^{16}$ H. Heinrich, K. Lischka, H. Sitter, and M. Kriechbaum, Phys. Rev. Lett. 35, 1107 (1975); H. Sitter, K. Lischka, and H. Heinrich, Phys. Rev. 16, 680 (1977).

${ }^{17}$ J. R. Burke, Jr., R. S. Allgaier, B. B. Houston, Jr., J. Babiskin, and P. G. Siebenmann, Phys. Rev. Lett. 14, 360 (1965); J. R. Burke, Jr., B. Houston, H. T. Savage, J. Babiskin, and P. G. Siebenmann, in Proceedings of the International Conference on the Physics of Semiconductors, Kyoto, 1966 [J. Phys. Soc. Jpn. Suppl. 21, 384 (1966)].

${ }^{18}$ H. T. Savage and B. Houston, Bull. Am. Phys. Soc. 15, 311 (1970).

${ }^{19}$ L. Liu and G. Bastard, Phys. Rev. B 25, 487 (1982).

${ }^{20}$ R. E. Watson and A. J. Freeman, Phys. Rev. 152, 566 (1966).

${ }^{21}$ W. Baltensperger and A. M. de Graaf, Helv. Phys. Acta 33, 
881 (1960).

${ }^{22}$ S. Lara, R. Moreira Xavier, and C. A. Taft, J. Phys. Chem. Solids 38, 795 (1977).

${ }^{23}$ J. R. Cullen, E. Callen, and A. H. Luther, J. Appl. Phys. 39, 1105 (1968).

${ }^{24} \mathrm{C}$. Kittel, in Solid State Physics: Advances in Research and Applications, edited by F. Seitz and D. Turnbull (Academic, New York, 1968), Vol. 22, p. 1.

${ }^{25}$ D. C. Mattis, The Theory of Magnetism (Springer, Berlin, 1981).
26J. Ginter, L. Świerkowski, and J. Kossut, Phys. Status Solidi B 96, 735 (1979).

27J. A. Mydosh and G. J. Nieuwenhuys, in Ferromagnetic Materials, edited by E. P. Wohlfarth (North-Holland, Amsterdam, 1980), Vol. 1, p. 71.

${ }^{28}$ J. Ruvalds, F. Brosens, L. F. Lemmens, and J. T. Devreese, Solid State Commun. 23, 243 (1977).

${ }^{29}$ P. G. de Gennes, J. Phys. Radium 23, 630 (1962).

${ }^{30}$ W. J. M. de Jonge, T. Story, H. J. M. Swagten, and P. J. T. Eggenkamp, Europhys. Lett. (to be published). 